\title{
Critical Success Factors for Migrating to OSS-on-the-Desktop: Common Themes across Three South African Case Studies
}

\author{
Daniel Brink, Llewelyn Roos, James Weller and Jean-Paul Van Belle \\ Department of Information Systems, University of Cape Town \\ Private Bag, Rondebosch 7701, South Africa \\ jvbelle@commerce.uct.ac.za \\ www.uct.ac.za
}

\begin{abstract}
This paper investigates the critical success factors associated with the migration from proprietary desktop software to an open source software (OSS) desktop environment in a South African context. A comparative case study analysis approach was adopted whereby three organisations that have migrated to desktop OSS were analysed. For diversity, one case study each was drawn from government, private industry and the educational sector. Most of the findings agree with those in the available literature though there are notable differences in the relative importance of certain factors.
\end{abstract}

\section{Introduction}

The market share of OSS is growing significantly [1]. Some of the factors contributing to the growth include the rising prices of Microsoft products, increased availability of OSS, increased quality and effectiveness of desktop OSS software and the drive for open standards in organisations and governments [1].

Correspondingly, there has been an increased interest and awareness of OSS in South Africa. Whilst OSS has been accepted as a viable alternative to proprietary software (PS) in the network server market for some time, desktop usage of OSS still remains fairly limited [2]. The high PS licensing and computer hardware costs in South Africa relative to the developed countries in combination with the several other perceived advantages of OSS have prompted several OSS on the desktop pilot projects in the education, public and private sectors. However, because no comprehensive follow-up study has been conducted to investigate the long-term outcomes of these projects, little is known about the true benefits and problems associated with the migration to desktop OSS in South Africa, why these migrations were undertaken in the first place, or how to successfully go about migrating to desktop OSS [3]. This lack of knowledge was the inspiration for our research.

This research seeks to uncover the critical success factors associated with the migration to desktop OSS in a South African context by means of a multiple case study analysis approach. In this paper, desktop OSS comprises those OSS applications that are utilised by every day users to perform daily work tasks. Hopefully future migrations to desktop OSS may be assisted by taking cognisance of the critical success factors found in this research.

Please use the following format when citing this chapter:

Brink, D., Roos, L., Weller, J., and Van Belle, J.-P., 2006, in IFIP International

Federation for Information Processing, Volume 203, Open Source Systems, eds. Damiani, E., Fitzgerald, B., Scacchi, W., Scotto, M., Succi, G., (Boston: Springer), pp. 287-293 


\section{Previous Research}

A number of researchers have proposed methodologies or guidelines to implement Desktop OSS. The Lachniet [4] framework focuses on the pre-work which needs to be done before migrating to desktop OSS in a corporate environment. The premigration tasks are divided into three sections: administrative tasks, application development tasks and information technology tasks with each grouping sub-divided in a number of tasks.

The methodology suggested by the Wild Open Source Inc consultants [5] consists of three phases: the planning phase, design phase and implementation phase. Here, the users are not involved in the migration process and only receive training at the very end of the migration. Also, their methodology does not specify how the migration should be done, merely stating that it should be planned and documented.

NetProject proposes an OSS Migration Methodology which divides the migration into following five exercises: data gathering and project definition phase, justification for the migration and estimate migration costs, Piloting phase, complete roll-out and implementation monitoring against the project plan [6].

\section{Research Methodology}

The main research question which is explored in this paper is "What are the critical success factors for migrating to desktop OSS, particularly in a South African context?" Because of the nature of the question, the research is exploratory as opposed to explanatory in nature. A qualitative approach was chosen as appropriate for this research because it enables researchers to make sense of a situation and gain a much richer understanding of a process or experience, via the analysis of people's spoken words and or writings, than a quantitative approach permits. The research methodology adopted was case study research because of its ability to provide subtle yet deep insights into social phenomena surrounding Information Systems [7]. Three case studies were conducted; one in each of the government, private (business) and education sectors. Thematic analysis was utilised to analyse data obtained in the case studies. First, theme analysis was performed for each case study separately. In a second round the common themes relating to the critical success factors across all three case studies were extracted. Two data gathering instruments were used for this research; semi-structured interviews and document analysis. The semi-structured interviews were held with stakeholders from three different organisation levels: management level staff who was involved in the decision to migrate to desktop OSS; IT support staff who was involved in the migration process and/or support of the OSS; and users who experience the desktop OSS on a day-to-day basis. In addition, relevant document were also studied. 


\section{The Three Case Studies}

Three case studies were analysed. For diversity, these were drawn from three different sectors: government, private (business) and education.

\subsection{Case Study 1: Novell SA}

In 2003, Novell acquired SUSE, the developer of a popular Linux distribution, which would become the new platform for their product range. In 2005, a corporate decision was taken to migrate Novell globally towards OSS. Being a relatively small office with a young management team, Novell SA made the decision to blaze the trail for desktop migration and adopt the Linux desktop across the country and employees were switched directly from Microsoft Windows to Linux overnight. Today there are no Microsoft Windows machines in Novell SA, except for a few software engineers who keep a copy for application compatibility testing. The rapid adoption of Novell Linux Desktop across South Africa did cause some problems as expected, but they were quickly resolved as the software matured.

\subsection{Case Study 2: Mossel Bay Municipality.}

The Municipality of Mossel Bay, a coastal town located in the Western Cape endeavoured to migrate to desktop OSS after software licensing issues, related to Microsoft products in use, were raised by the Business Software Alliance (BSA). In response to the letters from the BSA and the threat of possible legal issues, the majority of the PCs in the Financial Department were migrated to Linux, as the users use primarily network-based financial systems, email and spreadsheets. Although a number of different productivity suites and Linux versions were tested, the final configuration consisted of OpenOffice running on Novell SUSE Linux. In order to run Linux with a Graphical User Interface (GUI) some of the PCs had to be upgraded. The total duration of the migration to OSS was about 3 months. The IT Manager classifies the migration as a success, but admits that it "did not solve all the problems; at this point in time [the Municipality is] still under-licensed".

\subsection{Case Study 3: Pinelands High School}

Pinelands High School (hereafter referred to as "the school" or "Pinelands") is a secondary education school in the Cape Town metropolitan area. Their catalyst for getting OSS software onto more desktops at Pinelands was the failure of the school's intercom and announcements system in January 2004. The amount required to completely replace the existing system was considered exorbitant by school management. At this point, the IT Manager came up with the idea to replace the announcements system with a computer-based one. The new computer-based 
announcements system, affectionately known as IntraCom, is a web-based application running on the school's intranet. Furthermore, staff can access the internet and email, as well as produce text documents, spreadsheets and presentations, using OpenOffice.org, all from the comfort of their own classroom.

\section{Common Emergent Themes across the Case Studies}

Each of the cases was first analysed on its own, with a number of emergent themes emanating from each case. The emergent themes exposed in each of the three cases analysed were then synthesised into a group of themes found to be both common and of importance across all three cases. Based upon these derived themes, Critical Success Factors (CSFs) for the migration to desktop OSS are then presented.

\subsection{Financial Motivating Factor}

In all three cases, the primary reason for migrating to desktop OSS was financially related. For Novell, it was a case of moving into an emerging market and gaining competitive advantage, in order to secure better long-ierm financial performance. For the Mossel Bay Municipality, the move was required in order to avoid a large fine from the $B S A$ or the expense of purchasing and maintaining a large number of Microsoft Windows licenses. For Pinelands, desktop OSS provided a cheap alternative to replacing the school's defunct intercom system. Thus, consistent with the literature [8], financial reasons seemed to be one of the key drivers when deciding to migrate to desktop OSS.

\subsection{Top Management Support}

In all cases, the migration project was strongly supported by top management. In the case of Novell, the project was championed globally; in Mossel Bay, the project was endorsed by Council and finally, in the Pinelands case, the school's governing body fully backed the migration. This is essential since a project which introduces such a drastic degree of change into the organisation inevitably meets with fierce resistance from those affected, and migration to desktop OSS is no exception.

\subsection{User Awareness and Communication}

Although no strong evidence is available from the analysis of the Pinelands High School case study, both the Novell and Mossel Bay Municipality cases provide evidence of the value of facilitating good communication between management and users, as well as the creation of user awareness early on the migration process. Novell created a large and informative internal website called OpenZone, containing information on the why, how and when of the project, including discussion forums where participation was incentivised. Additional, a local advocate/expert was identified in each department. Mossel Bay Municipality also instigated significant user awareness and communication measures. 


\subsection{Detailed Planning, Analysis and Testing}

In all three cases, the importance of thorough planning, as well as the effects of improper planning, was apparent. In the Novell case, extensive planning and analysis was conducted before the migration commenced. This included the discovery of affected users and application dependencies, the construction of an application and hardware inventory, a list of new application requirements, as well as the derivation of a detailed time frame and list of objectives. With the Mossel Bay Municipality, the first rollout attempt was a failure due to technical problems, caused by not fully testing the system before handing it over to users, not correctly investigating the hardware requirements of some of the software installed, as well as installing office productivity software that did not meet user requirements. In Pinelands, several unique technical and functional requirements were overlooked due to the "one size fits all" approach of the tuXlabs implementation plan.

\subsection{Training}

All of the organisations studied conducted user training as part of the migration process but their approach, attitude and methods towards training all varied significantly. The best training practices were evident at Novell. By acknowledging the fact that individuals possess different styles and paces at which they learn, a flexible training programme was implemented, using web-based interactive tutorials, watching webcasts and training documentation. This fosters an active learning style. In contrast, Mossel Bay Municipality utilised the passive approach initially. Introductory seminars for the Linux desktop and OpenOffice.org were held, but some users did not attend. As a result, the trainer spent a lot of time running around in the office assisting users with problems, once the migration was rolled out. The training received at Pinelands High School was found to be of little use and once, again, attempts to conduct passive training sessions were not particularly successful.

\subsection{Pilot Project and Partial Migration}

[4] argues for implementing a pilot migration project with only a select group of users. Analysis of the three cases revealed a general agreement amongst the IT Managers interviewed with the arguments presented in the literature. Whilst Novell managed to leverage its resources to enable a complete migration, both Mossel Bay Municipality and Pinelands High School found it impossible to migrate fully to desktop OSS. This was due to the presence of "mission critical" legacy applications that no suitable OSS alternative could be found for. In both cases, this meant that Microsoft Windows had to be retained on some of the organisational computers.

\subsection{Support}

In all cases, the importance of post-implementation support was highlighted. In the case of Novell, a 24 hours, 7 days per week helpdesk is available, along with numerous online support websites, to assist users in resolving problems. Furthermore, a large base of in-house support is available. Mossel Bay municipality outsources a large part of its desktop OSS support, as only the IT Manager possesses 
detailed in-house support skills. Furthermore, the majority of interviewees felt that the level of support required at Mossel Bay was not being met. At Pinelands High School, support also remains a problem and forms a large portion of the IT budget.

\section{Conclusion and Summary}

This research aimed to identify critical success factors for projects dealing with migration towards OSS-on-the-desktop, in a developing world context. This was done by an in-depth analysis of three case studies.

Consistent with the literature, the main driver for deciding to migrate to desktop OSS was the promise of financial benefits, such as decreased license costs and the ability to redistribute funds that would have been spent on software licenses to other areas. No evidence of any of the migrations being motivated by political or social responsibility factors could be found. There was also mention of intangible benefits, such as the freedom from vendor lock-in and the ability to customise the software should one wish to do so. Other supposed benefits identified in the literature, such as improved security, did not appear to be important to the organisations studied.

The problems of legacy applications preventing total migration, user resistance and high support costs were identified in all of the case studies. Problems related to training, specifically the general perception of non-usefulness of training and the lack of a hands-on, practical approach to training, were identified.

Future research should investigate to which extent these findings can be generalised to other contexts. The researchers are currently using the findings as a basis for the development of a more comprehensive migration methodology.

\section{References (abbreviated list)}

[1] Wheeler, D. Why Open Source Software / Free Software (OSS/FS, FLOSS, or FOSS)? Look at the Numbers! (2 April 2005) http://www.dwheeler.com/oss_fs_why.html.

[2] Prentice, S. \& Gammage, B. Enterprise Linux: Will Adolescence Yield to Maturity? Gartner Symposium/ITxpo (2005).

[3] Bruggink, M. Open Source Software: Take It or Leave It? The Status of Open Source Software in Africa, International Institute for Communication and Development, (16 June 2003) http://www.ftpiicd.org/files/research/reports/report16.doc.

[4] Lachniet, M. Desktop Linux Feasibility Study Overview, (20 August 2004), http://lachniet.com.desktoplinux.

[5] Wild Open Source. Steps to Take when Considering a Linux Migration, (25 August 2005), http://www.wildopensource.com/technology_center/steps_linux_migration.php

[6] Netproject. IDA OSS Migration Guidelines, (29 August 2005), http://www.netproject.com/docs/migoss/v1.0/methodology.html.

[7] Walsham, G. 'Interpretive Case Studies in IS Research: Nature and Method', European Journal of Information Systems, 4(2), 74-81 (1995). 
[8] Gardiner, J.; Healey, P.; Johnston, K. \& Prestedge, A. The State of Open Source Software (OSS) in South Africa, Unpublished Technical Report, University of Cape Town, 2003. 